\title{
Left coronary artery dissection: an unusual presentation
}

\author{
GRAHAM F ADKINS, * RICHARD H STEELE $\dagger$ \\ From the ${ }^{\star}$ Department of Pathology, Royal Brisbane Hospital, Herston; and the $\dagger$ Department of Pathology, \\ University of Queensland, Australia
}

SUMMARY Primary dissecting aneurysm of the left main stem coronary artery is a rare cause of coronary arterial occlusion that usually presents as sudden death or myocardial infarction in a young woman. A 32 year old woman with no signs of cardiac disease who died as the result of a right cerebral infarction was found to have coronary dissection and myocardial infarction at necropsy.

\begin{abstract}
Dissection of a coronary artery may occur secondary to the extension of an aortic dissection, in association with coronary angiography, ${ }^{12}$ or as a complication of cardiac operations. ${ }^{3}$ Spontaneous primary dissection of a coronary artery is an uncommon cause of death that is seen predominantly in young women. It was first described in 1931 in the right coronary artery of a 42 year old woman. ${ }^{4}$ Since then most case reports have shown that the dissection affects the anterior descending branch of the left coronary artery. Twelve cases of left main stem coronary artery involvement have been reported and in most of them dissection included the left anterior descending and/ or left circumflex arteries as well. ${ }^{5}$ Our case is unusual in that the dissection was restricted to the left main stem coronary artery and did not extend into either of its two branches.
\end{abstract}

\section{Case report}

A white woman aged 32 had a history of asthma as a child. She had had three uneventful pregnancies; her youngest child was three years old. She was on no medication, was not taking oral contraceptives, and smoked up to 20 cigarettes a day.

One week before admission she and her family ate a meal of freshly caught prawns. Soon after this she experienced epigastric discomfort, vomiting, and diarrhoea; other members of the family were

Requests for reprints to Dr R H Steele, Department of Pathology, University of Queensland, Herston Road, Herston, Queensland 4006, Australia. unaffected. Her illness persisted for two days and she attributed it to food poisoning. The temporal coincidence of these symptoms with the age of the dissection, however, suggests that they may have been produced by vagal or sympathetic stimulation caused by the dissection. After this episode she remained well for four days. On the day of admission she noted the sudden onset of paraesthesia and weakness on the left side of her body after she had eaten her evening meal. She was transferred to the casualty department of the Royal Brisbane Hospital where she was found to be conscious but drowsy. Blood pressure was $80 / 60 \mathrm{~mm} \mathrm{Hg}$. Physical examination showed a left sided decrease in muscle tone and loss of muscle power associated with hyperreflexia. The remainder of the examination was unremarkable. In particular the pulse rate was 72 beats per minute and regular, the heart sounds were normal, and no murmurs were heard.

Investigations showed widespread depression of the ST segment in leads II, III, and aVF on electrocardiography (Fig. 1). The only biochemical abnormality detected was a lactate dehydrogenase activity of 542 units/litre (normal range 125-220). Chest and skull $x$ rays were reported to show no abnormality. A computerised axial tomography head scan showed a right sided low density lesion that was consistent with a large infarct in the area of distribution of the right middle cerebral artery. There was no evidence of subarachnoid haemorrhage. No underlying cause was found for the right cerebral infarct. Her neurological state deteriorated and she died two days after admission. 

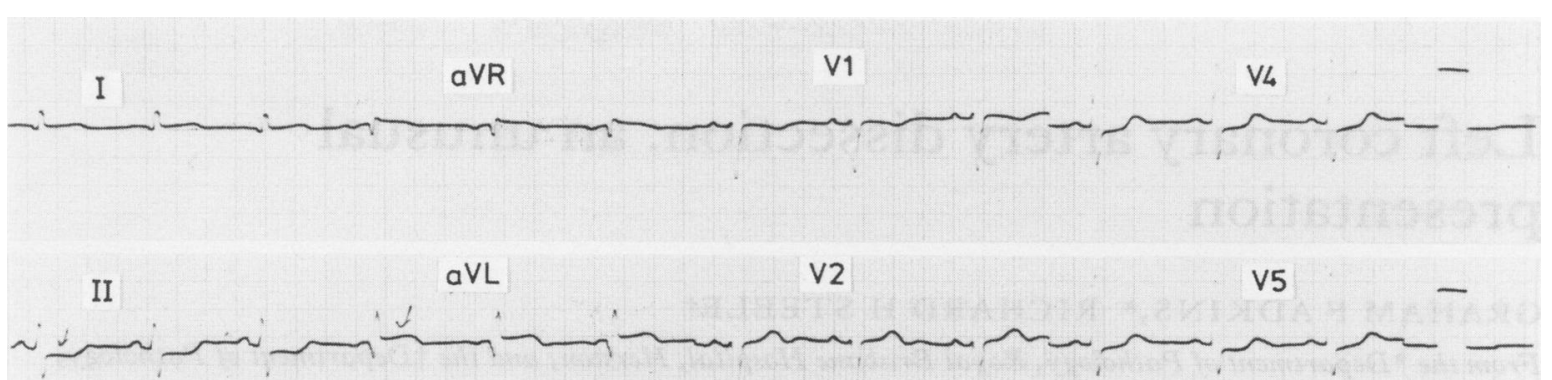

Fig. 1 Electrocardiogram obtained at admission one week after first symptoms. It was not considered to be diagnostic of heart disease.

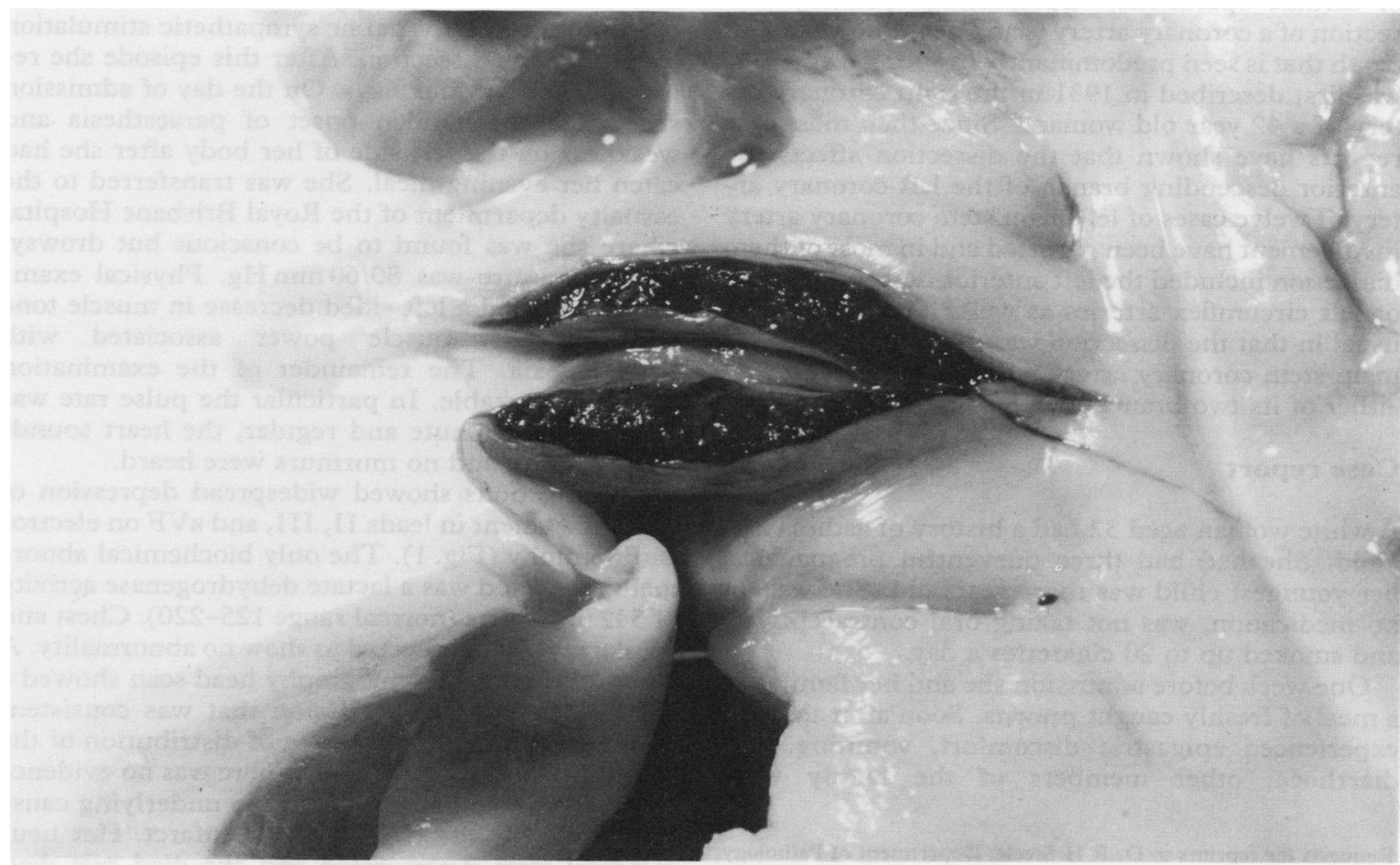

Fig. 2 The first part of the left coronary artery opened to show the dissecting aneurysm. 


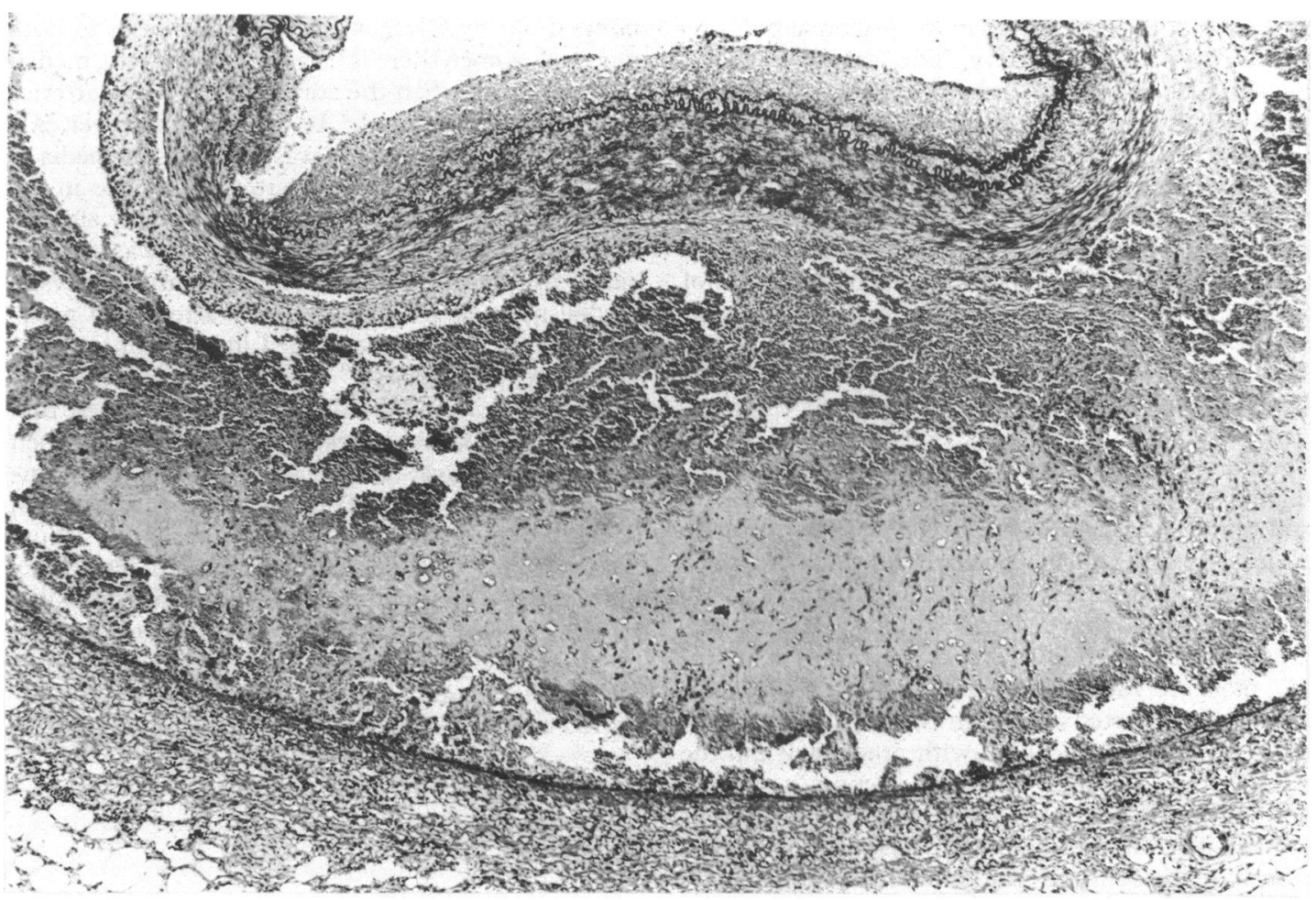

Fig. 3 Photomicrograph of wall of the left coronary artery showing the dissection in the outer third of the media. Verhoff van Gieson; original magnification $\times 20$.

\section{Pathology}

External examination at necropsy showed the body of a young adult female (height $1.65 \mathrm{~m}$, weight $68 \mathrm{~kg}$ ). Her fingers were unusually long when compared with her body build and gave the subjective impression of arachnodactyly. No other features suggestive of Marfan's syndrome were found, although they were specifically sought. The heart was of normal size and weighed $320 \mathrm{~g}$. Subendocardial to full thickness infarction affected the anterior one third of the interventricular septum and the anterior and lateral portions of the free wall of the left ventricle. The region infarcted was in the distribution area of the left coronary artery. The coronary arteries arose normally from the aorta and had a normal anatomical distribution with the left main stem coronary artery bifurcating and giving rise to normally sized left anterior descending and left circumflex branches.

There was a dissecting aneurysm of the left main stem artery (Fig. 2) which had resulted in total occlusion of the vessel lumen. This dissection affected the length of the left main stem but did not involve the aorta or either of the two branches of the left coronary artery. No internal tear was identified.

Examination of the remainder of the heart showed mural thrombi in the left atrial appendage and in the left ventricle overlying the area of infarction. The right coronary artery, aorta, and its branches and large muscular arteries throughout the body appeared to be macroscopically normal. An occlusive thrombus, consistent with an embolus from the left atrium or ventricular wall, was found in the distal right internal carotid artery and there was recent infarction of the right cerebral hemisphere in the area of distribution of the right middle cerebral artery.

Histological examination showed a circumferential dissection of the left main stem coronary artery with separation of the outer one third of the media from the adventitia and evidence of early organisation of the clotted blood (Fig. 3). There were a few lymphocytes and eosinophils in the adventitia, but there was no evidence of vasculitis. Diffuse intimal thickening with abundant smooth muscle cells and some elastin was seen in the left main stem coronary artery, but there were no features of atherosclerosis and no evidence of intimal disruption. 
Similar changes were noted in the proximal portions of the right coronary artery. The arterial media of both vessels contained recognisable small pools of Alcian blue positive mucopolysaccharide material. Examination of the ascending and descending portions of the thoracic aorta showed prominent accumulations of mucopolysaccharide within the lamellar units and this was associated with fragmentation and focal loss of elastic fibres. No evidence of dissection was found in the root of the aorta adjacent to the left coronary ostium.

\section{Discussion}

Spontaneous dissection of a coronary artery unrelated to coronary angiography, cardiac surgery, and blunt chest trauma is rare but well recognised. At least 75 cases have been reported since the original description in $1931,{ }^{5}$ and several reviews of the published cases have demonstrated similar background features. ${ }^{56}$ Most patients are women (60 of 75 ), most are young (mean age 37-42 years), and 19 of 60 cases reported in women occurred peripartum. Apart from this association with pregnancy and the puerperium, coronary artery dissection is usually not associated with any other clinical condition or pre-existing disease state. One case has been previously reported in association with arachnodactyly. Though, as in our case, the patient had no other stigmata of Marfan's syndrome. ${ }^{7}$ In most cases the left anterior descending coronary artery is dissected but any of the main coronary arteries may be affected. Twelve cases of dissection of the left main stem coronary artery have been reported. ${ }^{5}$ Many of the cases have shown extension of the dissection into the left anterior descending and or left circumflex branches but this feature was not present in our case.

Clinically most cases present with sudden death or with symptoms and signs of myocardial infarction. Our case is unusual in that the probable episode of dissection was believed by the patient to be a bout of food poisoning and she did not seek medical assistance. She then remained well and symptom free for four days until she presentated with an acute cerebral infarct, presumably the result of embolus from the left ventricle. Even on admission there was no clinical sign of heart disease and the electrocardiographic changes were regarded as not specific.

The histological changes in the coronary arteries and aorta are of interest. Cystic medial necrosis (the pooling of acid mucopolysaccharide material) has been described in 25 of the 66 cases of spontaneous dissection examined microscopically. These included a woman in the post partum period, ${ }^{8}$ a woman who had not recently been pregnant, ${ }^{9}$ and an elderly man with alcoholic liver disease. ${ }^{10}$ As pointed out by Glasgow et al in many cases in both men and women there is no evidence of cystic medial necrosis, even when the aorta and coronary arteries are carefully examined. ${ }^{5}$ In our case, however, Alcian blue positive material was found in the media of the left main stem coronary artery as well as in the media of the proximal right coronary artery and the aorta. Fragmentation and loss of elastin was an associated feature in the aorta. The association of cystic medial necrosis in both coronary artery and proximal aorta has been mentioned in some of the previously reported cases, ${ }^{1011}$ though not in others.

Molloy et al and Malloch have emphasised that dissection should be suspected in young women with no previous history who present with unexpected circulatory collapse, myocardial ischaemia, or infarction, especially in the puerperium. ${ }^{912}$ Suspicion of the diagnosis may then lead to emergency investigation of the coronary arteries and possible life-saving cardiac operation. Unfortunately our patient had no definite symptoms or signs to indicate a cardiac disease and, as with so many previously reported cases, the diagnosis was made at necropsy.

\section{References}

1 Guss SB, Zir LM, Garrison HB, Daggett WM, Block PC, Dinsmore RE. Coronary occlusion during coronary angiography. Circulation 1975; 52: 1063-8.

2 Harrison LH, Gregg DL, Itescoitz SB, Redwood DR, Michaelis LL. Delayed coronary artery dissection after angiography. $f$ Thorac Cardiovasc Surg 1975; 69: 880-3.

3 Bulkley BH, Roberts WC. Isolated coronary artery dissection. F Thorac Cardiovasc Surg 1974; 67: 148-51.

4 Pretty HC. Dissecting aneurysm of coronary artery in a woman aged 42: rupture. $\mathrm{Br} \mathrm{Med} \mathcal{F} 1931$; i: 667.

5 Glasgow BJ, Tift JP, Alexander CB. Spontaneous primary dissecting coronary artery aneurysm. Am $\mathcal{f}$ Forensic Med Pathol 1984; 5: 155-9.

6 Brody GL, Burton JF, Zawadzki ES, French AJ. Dissecting aneurysms of the coronary artery. $\mathrm{N} \mathrm{Engl} \mathcal{F}$ Med 1965; 273: 1-6.

7 McKeown F. Dissecting aneurysm of the coronary artery in arachnodactyly. Br Heart $\mathcal{f} 1960$; 22: 434-6.

8 Jewett JF. Two dissecting coronary artery aneurysms postpartum. N Engl F Med 1978; 298: 1255-6.

9 Molloy PJ, Ablett MB, Anderson KR. Left main stem coronary artery dissection. Br Heart $\mathcal{f} 1980$; 43: 705-8.

10 Gibson WG, Reimer KA. Multiple coronary artery dissections in old age. Arch Pathol Lab Med 1980; 104: 419-21.

11 Claudon DG, Claudon DB, Edwards JE. Primary dissecting aneurysm of the coronary artery. Circulation 1972; 45: 259-65.

12 Malloch JA. Dissecting aneurysm of coronary artery. NZ Med F 1974; 79: 914-8. 